The Israeli Journal of Aquaculture - Bamidgeh, IJA_69.2017.1401, 7 pages

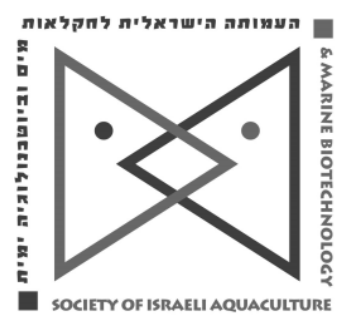

The IJA appears exclusively as a peer-reviewed on-line open-access journal at http://www.siamb.org.il. To read papers free of charge, please register online at registration form.

Sale of $I J A$ papers is strictly forbidden.

\title{
Rapid and Sensitive Detection of Vibrio natriegens in Portunus trituberculatus with the Loop-Mediated Isothermal Amplification Test
}

\author{
K.R. Bi ${ }^{1,2 *}$, Z.H. Lu ${ }^{1,2}$, B.L. Yan ${ }^{1,2}$, H. Gao ${ }^{1,2}$, J. Qian $^{1,2}$ \\ ${ }^{1}$ Huaihai Institute of Technology, Jiangsu Key Laboratory for Marine Biotechnology, \\ Co-Innovation Center of Jiangsu Marine Bio-industry Technology, Lian Yungang \\ 222005, P.R. China \\ 2 The Jiangsu Provincial Platform for Conservation and Utilization of Agricultural \\ Germplasm, Nanjing 210014, P.R. China
}

Keywords: Portunus trituberculatus; Vibrio natriegens; loop-mediated isothermal amplification (LAMP)

\begin{abstract}
In order to detect Vibrio natriegens in Portunus trituberculatus, a loop-mediated isothermal amplification (LAMP) method was developed and evaluated. In this study LAMP primers were designed to correspond to the gyrB gene sequences. With Bst DNA polymerase, the LAMP assay was completed within 100 min at $65^{\circ} \mathrm{C}$ in a water bath. Amplification products were observed directly with agar gel or with the naked eye after the addition of SYBR Green I. The sensitivity of the LAMP assay for the detection of $V$. natriegens is about $1.32 \times 10^{-2} \mathrm{fg} / \mathrm{mL}$ DNA template, whereas using duplex PCR the detection of $V$. natriegens was possible up to $13.2 \mathrm{pg} / \mathrm{mL}$ DNA template. There were no cross-reactions with other Vibrio strains indicating a high specificity of the LAMP. The novel LAMP assay in this study can be used as a valuable, rapid, and sensitive detection tool for the detection of $V$. natriegens both in the laboratory and for use in commercial aquaculture.
\end{abstract}

\footnotetext{
* Corresponding author. Tel.: +86-518-85895252, fax: +86-518-85895252, e-mail: bikeran@126.com
} 


\section{Introduction}

The swimming crab, Portunus trituberculatus (Crustacea: Decapoda: Brachyura), has become an important marine fishery and aquaculture species. It comprises a large proportion of crab aquaculture in China, with the total yield exceeding 100,000 tons in 2011 (Lv et al., 2014). However, with the rapid development of intensive crab culture, the sustainability of the crab aquaculture industry has been threatened by outbreaks of diseases, particularly those caused by bacteria in the genus Vibrio (Liu et al., 2007; Yan et al., 2010; Wan et al., 2011; Zhang et al., 2014; Bi et al., 2016).

$V$. natriegens is a Gram-negative, assumed to be non-pathogenic halophile marine bacterium (Payne et al., 1961). However, recent studies have showed that $V$. natriegens is pathogenic to aquatic animals and mortality rate was greater than 90\% (Bi et al., 2016; Deng et al., 2004; $\mathrm{Li}$ et al., 2009). Disease has been difficult to control because $V$. natriegens has the fastest growth rate of any known organism, with a reported doubling time of $<10 \mathrm{~min}$ (Weinstock et al., 2016). Therefore, there is an urgent need for in situ detection of $V$. natriegens, and the method should be fast, sensitive, reliable, easy to carry out, and results easily seen.

Loop-mediated isothermal amplification (LAMP) was first reported in 2000 (Notomi et al., 2000) and has since been used in various aspects of nucleic acid research, and in clinical application as an ideal tool for diagnosis (Dhama et al., 2014). The most innovative features of LAMP, is the simplicity of its protocol, and overall low cost of application when compared to other pre-existing molecular techniques, such as Nucleic Acid Sequence-Based Amplification (NASBA), Self-Sustained Sequence Replication (3SR), Standard Displacement Amplification (SDA), Rolling Circle Amplification (RCA), and most prominently Polymerase Chain Reaction (PCR) (Umar et al., 2015). In aquaculture, LAMP has been used widely for Vibrio detection in aquatic animal disease. It shows high specificity, sensitivity, and rapidity under isothermal conditions with the presence of Bst DNA polymerase (Wang et al., 2017; Zhou et al., 2016).

In this study, we developed an assay based on the LAMP technique for the detection of $V$. natriegens in $P$. trituberculatus, and investigated its sensitivity, specificity, and application potential in swimming crabs. Our results indicated that by using the LAMP method we identified $V$. natriegens rapidly and accurately. This method will assist in the detection of $V$. natriegens in swimming crab farms.

\section{Materials and Methods}

Several hundred swimming crabs were collected from farms located in Lianyungang City of Jiangsu Province, China. V. natriegens, V. parahaemolyticus, V. harveyi, V. alginolyticus, and V. vulnificus were previously isolated from swimming crabs and shrimp in our laboratory.

DNA extraction. All Vibrio strains were cultured on ZoBell's marine $2216 \mathrm{E}$ agar for $24 \mathrm{~h}$ or $48 \mathrm{~h}$ at $28^{\circ} \mathrm{C}$. Genomic DNA from these strains was extracted using the EasyPure Genomic DNA Kit (Beijing TransGen Biotech Co., Ltd. Beijing, China) according to the manufacturer's protocol. Briefly, the Vibrio cells that were cultured in $2 \mathrm{ml}$ ZoBell's marine 2216E medium were harvested by centrifugation at $15,700 \mathrm{~g}$ for $1 \mathrm{~min}$ at $4^{\circ} \mathrm{C}$. The supernatant was discarded and lysis buffer was added to the pellets and incubated at $55^{\circ} \mathrm{C}$ for $15 \mathrm{~min}$. The lysate was treated with RNase $A$ $(20 \mathrm{mg} / \mathrm{mL})$ and the genomic DNA was extracted. DNA quality was assessed by electrophoresis on $1.0 \%$ agarose gels and DNA concentration was measured using a NanoDrop 2000 (Thermo Scientific, USA).

Primers designed. Based on the gyrB gene sequence (accession numbers: KC912686.1), the primer of LAMP was designed using Primer Explorer V5 software (http://primerexplorer.jp/e/v5_manual/index.html). A forward inner primer (FIP), a reverse inner primer (BIP), and two outer primers (F3 and B3) were chosen and synthesized by Sangon Biotech (Shanghai) Co., Ltd. Information relating to the primers is shown in Table 1 and Figure 1 . In addition, 16S rDNA primer (27f/1942r) (Bi et al., 2016) and dulplex PCR primer (data not shown) were used to ascertain the specificity and sensitivity of LAMP assays.

Table 1. Primer names and sequences of LAMP test.

\begin{tabular}{cc}
\hline Primer Name & Sequences $\left(5^{\prime}-3^{\prime}\right)$ \\
\hline F3 & CAGCACGTGCTCGTGAAC \\
B3 & GGATCTACCTTCAGCGGC \\
FIP & AGCAAGTTTGCCGGAAGGC-TTT-AAGCTCGTGAAATGACTCGT \\
BIP & ACATTGTGGAGGGTGACTCTGC-TIT-GCCTGGTTCTTACGGTTACG \\
\hline
\end{tabular}




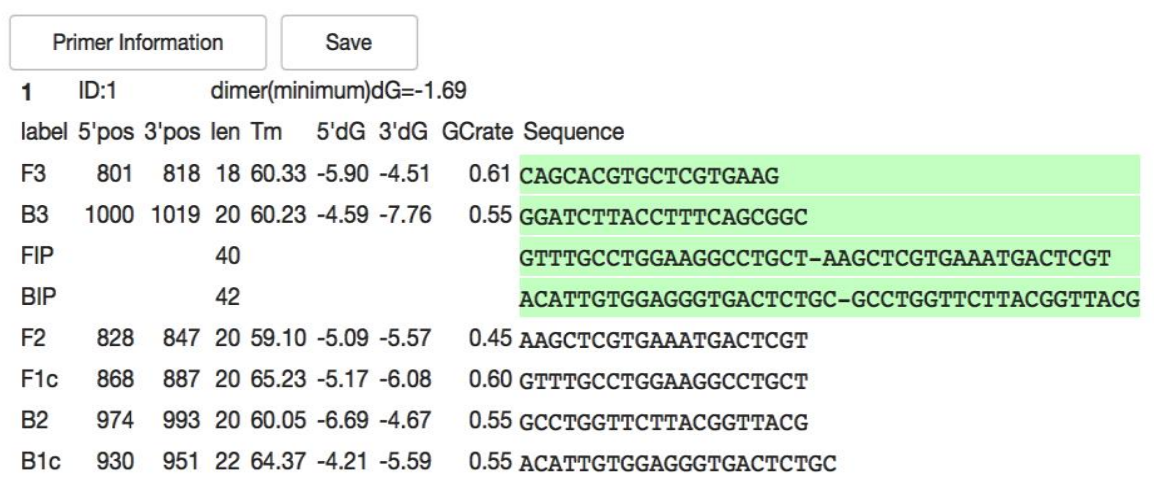

Fig. 1. The primers information of gyrB-LAMP.

Optimization for LAMP. In order to optimize the amplification system of LAMP assay, PCR experiments were carried out in a $25 \mu \mathrm{l}$ reaction mixture containing $10 \times$ Bst-DNA Polymerase Buffer, $25 \mathrm{mmol} / \mathrm{L} \mathrm{MgSO}_{4}, 10 \mathrm{mmol} / \mathrm{L}$ dNTP mixture, $5 \mathrm{~mol} / \mathrm{L}$ betaine, $10 \mu \mathrm{mol} / \mathrm{L} \mathrm{F3}, \mathrm{B} 3$, FIP and BIP, template DNA and $8000 \mathrm{U}$ Bst DNA polymerase (NEB, Beijing, China). Reaction time was optimized by incubating the mixture for $45,60,90$, and $120 \mathrm{~min}$ at $65^{\circ} \mathrm{C}$, while reaction temperature was optimized by incubating the mixture at $60,63,65$, and $66^{\circ} \mathrm{C}$ for $60 \mathrm{~min}$. Concentrations of betaine and $\mathrm{Mg}^{2+}$ ion in the LAMP reaction solutions were optimized using a series of concentrations from 1-6 mol/L and from 1-5 mol/L, respectively. Reaction was terminated by heating to $80^{\circ} \mathrm{C}$ for $10 \mathrm{~min}$. The LAMP products were detected by agarose gel electrophoresis and naked-eye observation after the addition of SYBR Green I (Beijing TransGen Biotech Co., Ltd. Beijing, China).

Specificity of LAMP. Four other marine pathogenic Vibrios, V. parahaemolyticus, V. harveyi, $V$. alginolyticu, and $V$. vulnificus, were used to investigate LAMP specificity. DNA templates of each strain were prepared by the method described in "DNA extraction". The template DNA was subjected to both LAMP and 16S rDNA PCR amplification. Each sample was tested three times with LAMP method.

Sensitivity of LAMP. In order to determine the sensitivity of LAMP assay, the DNA from $V$. natriegens $(132 \mu \mathrm{g} / \mathrm{mL})$ was serially diluted ten times and $1 \mu \mathrm{L}$ of each dilution was used as a template for the LAMP and dulplex PCR assays. Dulplex PCR was performed in a $25 \mu \mathrm{L}$ volume of reaction mixture containing $13 \mu \mathrm{L} 2 \times$ Master Mix, $1 \mu \mathrm{L} 10 \mu \mathrm{mol} / \mathrm{L}$ vhh-F/vhh-R, $2 \mu \mathrm{L}$ tox-F/tox-R, $1 \mu \mathrm{L}$ genomic DNA, and $5 \mu \mathrm{L}$ ultrapure water. The program involved the initial denaturation of $5 \mathrm{~min}$ at $95^{\circ} \mathrm{C}, 30$ cycles of $30 \mathrm{~s}$ at $94^{\circ} \mathrm{C}, 45 \mathrm{~s}$ at $53.3^{\circ} \mathrm{C}, 1 \mathrm{~min}$ at $72^{\circ} \mathrm{C}$, and a final $10 \mathrm{~min}$ extension at $72^{\circ} \mathrm{C}$. PCR products were electrophoresed to verify the presence of the expected 308 and 526 bp bands.

LAMP application for rapid diagnosis. From hundreds of swimming crabs that were collected from farms located in Lianyungang City of Jiangsu Province, China, 90 swimming crabs were randomly chosen and were used to detect the existence or nonexistence of $V$. natriegens using LAMP assays. In addition, muscle from each crab was homogenized in $2 \mathrm{ml}$ saline. The homogenates were plated onto ZoBell's marine $2216 \mathrm{E}$ agar. These plates were incubated at $28^{\circ} \mathrm{C}$ for $24 \mathrm{~h}$. Single colony cultures were prepared in ZoBell's marine $2216 \mathrm{E}$ broth, and a drop of culture was placed on formvar-coated copper grids and fixed with $2.5 \%$ glutaraldehyde for $1 \mathrm{~min}$. After removal of excess fluid by blotting on filter paper, the wet residues were immediately covered with the $2 \%$ sodium phosphotungstate for 30-40 s, and then withdrawn in the same fashion. The grid was air-dried and was observed and photographed by a Hitachi 600-2A TEM.

\section{Results}

Optimized LAMP reaction. After optimization, the reaction mixture $(25 \mu \mathrm{L})$ contained $2.5 \mu \mathrm{L} 10 \times$ BstDNA Polymerase Buffer, $2 \mu \mathrm{L} 25 \mathrm{mmol} / \mathrm{L} \mathrm{MgSO}_{4}, 2.5 \mu \mathrm{L} 10 \mathrm{mmol} / \mathrm{L} \mathrm{dNTP}$ mixture, $5 \mu \mathrm{L} 5 \mathrm{~mol} / \mathrm{L}$ betaine, $0.4 \mu \mathrm{L} 10 \mu \mathrm{mol} / \mathrm{L}$ each of $\mathrm{F} 3$ and B3, $1.6 \mu \mathrm{L}$ each of FIP and BIP, $1 \mu \mathrm{L}$ template DNA, $8 \mathrm{U}$ Bst DNA polymerase, and $8 \mu \mathrm{L}$ ultrapure water. $65^{\circ} \mathrm{C}$ was found to be the optimal temperature as at this temperature LAMP products displayed clearer amplification of DNA than at other temperatures including 60,63 , and $66^{\circ} \mathrm{C}$ (data not shown). To determine the optimum duration of the LAMP assay, four different reaction times, 45, 60, 90, and $120 \mathrm{~min}$ were used in the LAMP reaction. Although we could detect well-formed bands at $60 \mathrm{~min}$, the reaction time was extended to $90 \mathrm{~min}$ to ensure positive detection of lower concentration templates in the system. 
Specificity of LAMP. When LAMP was carried out using the DNA template from V. natriegens, V. parahaemolyticus, V. harveyi, V. vulnificus, and $V$. alginolyticus, color change in the reaction tubes with $V$. natriegens genomic DNA could be seen (Fig. 2A) within a 60 min incubation period, and the specific ladder of multiple bands was produced by $1.5 \%$ agarose gel electrophoresis (Fig. 2B). In the reaction of $V$. parahaemolyticus, $V$. harveyi, $V$. alginolyticus, and $V$. vulnificus strains, there was no color change in the reaction tubes after a $60 \mathrm{~min}$ incubation period and the specific ladder of multiple bands was not produced by $1.5 \%$ agarose gel electrophoresis. This result demonstrated that the LAMP primers were specific to $V$. natriegens identification. At the same time, their DNA was amplified by PCRs primed by $27 \mathrm{f} / 1942 \mathrm{r}$ primer in order to indicate the existence of four another Vibrio strains genomic DNA. The DNA products were unambiguously observed in PCRs (Fig. 2C).
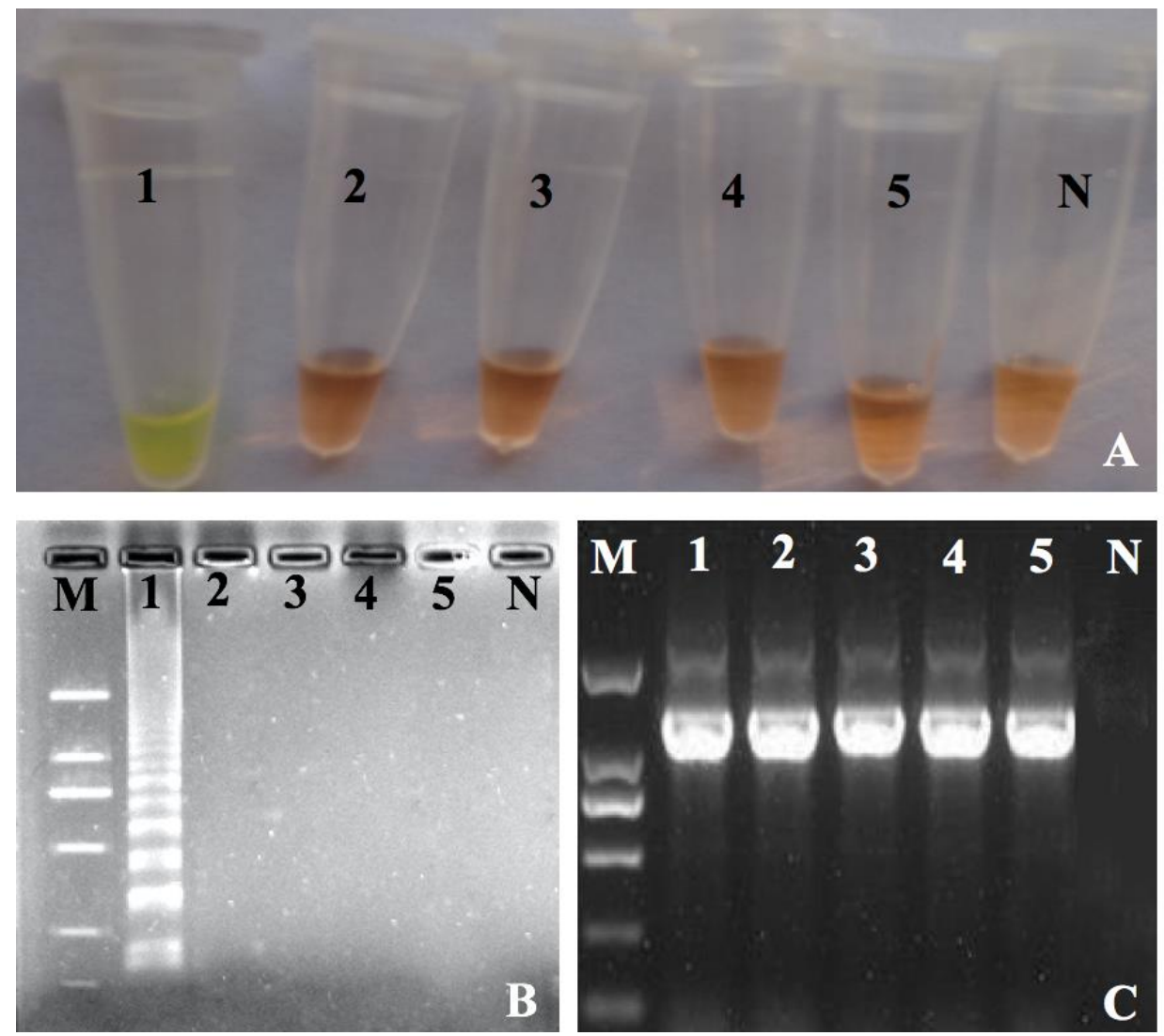

Fig 2. Specificity test of gyrB-LAMP and $16 S$ DNA PCR. (A) Color reaction with SYBR Green I; (B) Electrophoresis of gyrB-LAMP; (C) Electrophoresis of 16S DNA PCR. M: DL DNA marker; 1-5: V. natriegens, V. parahaemolyticus, V. harveyi, V. alginolyticus and $V$. vulnificus; N: negative control.

Sensitivity of LAMP. The reaction was tested using $1 \mu \mathrm{L}$ of the 10 -fold serial dilutions of $V$. natriegens DNA and compared to results from duplex PCR assay. The detection limit of the gyrB of LAMP was $1.32 \times 10^{-2} \mathrm{fg} / \mathrm{mL}$ (Fig. $3 A$ and $3 B$ ), and LAMP reaction followed by colorimetric analysis could be completed within 100 minutes. However, the detection limit of duplex PCR based on vhh and toxR genes was $13.2 \mathrm{pg} / \mathrm{mL}$, but the following gel electrophoresis required about $3 \mathrm{~h}$ for completion (Fig. 3C). 


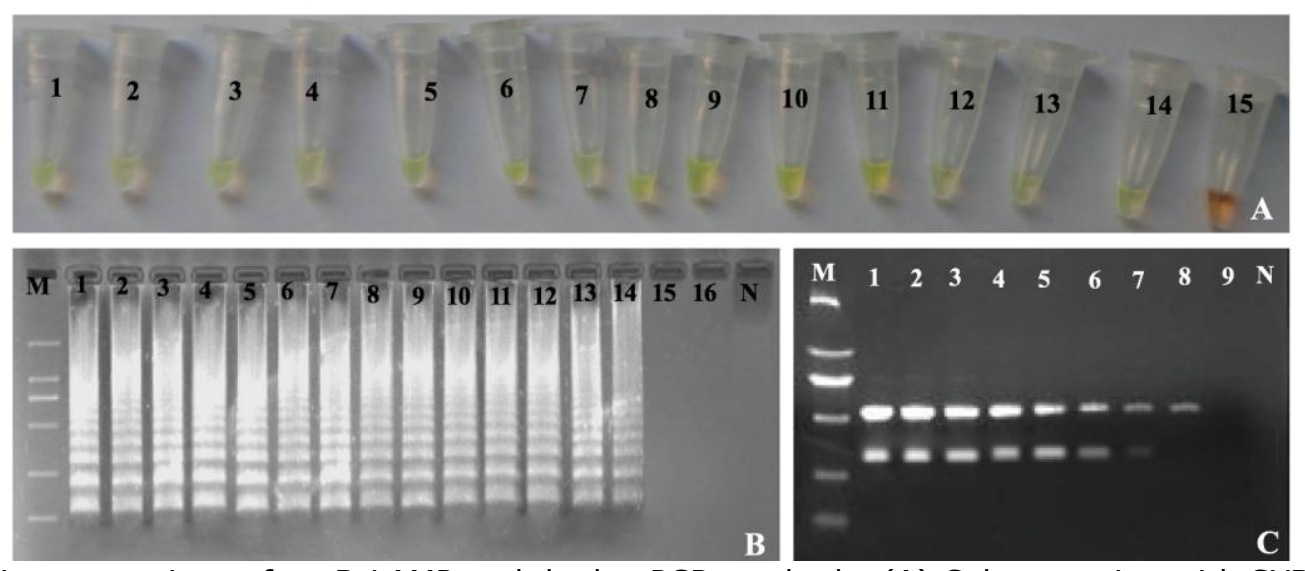

Fig 3. Sensitivity comparison of gyrB-LAMP and duplex PCR methods. (A) Color reaction with SYBR Green I; (B) Electrophoresis of gyrB-LAMP; (C) Electrophoresis of duplex PCR. M: DL DNA marker; 1-14: various dilutions of V. natriegens DNA template: $1.32 \times 10^{2} ; 1.32 \times 10^{1} ; 1.32 \times 10^{0} ; 1.32 \times 10^{-1} ; 1.32 \times 10^{-2} ; 1.32 \times 10^{-3} ; 1.32 \times 10^{-4}$; $1.32 \times 10^{-5} ; 1.32 \times 10^{-6} ; 1.32 \times 10^{-7} ; 1.32 \times 10^{-8} ; 1.32 \times 10^{-9} ; 1.32 \times 10^{-10} ; 1.32 \times 10^{-11} ; \mathrm{N}$ : negative control.

LAMP application for rapid diagnosis. Of the 90 swimming crabs tested, LAMP detected 34 crabs which were infected by $V$. natriegens (Fig. 4A and 4B). By comparison, in vitro culture of muscle homogenate, isolation and examination by transmission electron microscopy (TEM) by negative staining (Fig 5), $V$. natriegens isolates could be detected in only 18 crabs since the isolation technique could only detect living $V$. natriegens. In the muscle homogenate of the remaining 72 crabs, $V$. natriegens was not observed, even when incubation time was extended to one week. Therefore, the LAMP technique was more efficient, sensitive, and accurate than the in-vitro isolation procedure for the detection of the $V$. natriegens pathogen in swimming crabs.
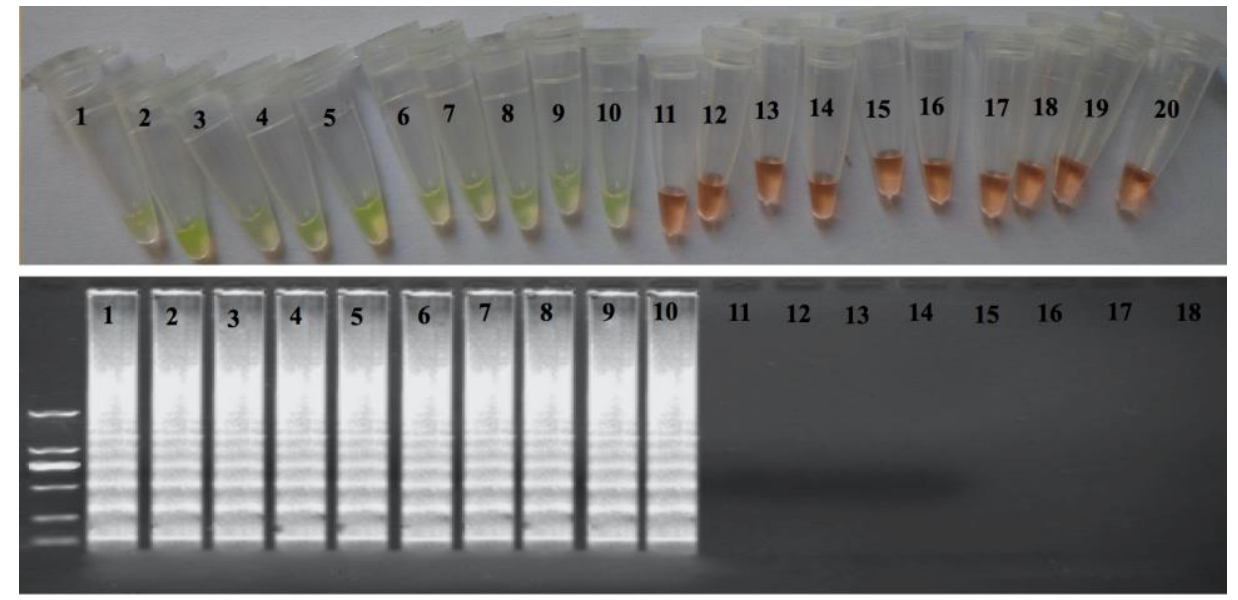

Fig 4 Application of gyrB-LAMP to detect $V$. natriegens from $P$. trituberculatus. (A) Color reaction with SYBR Green I; (B) Electrophoresis of gyrB-LAMP; lane1-10: positive results of gyrB-LAMP; Lane 11-20: negative results of gyrB-LAMP.

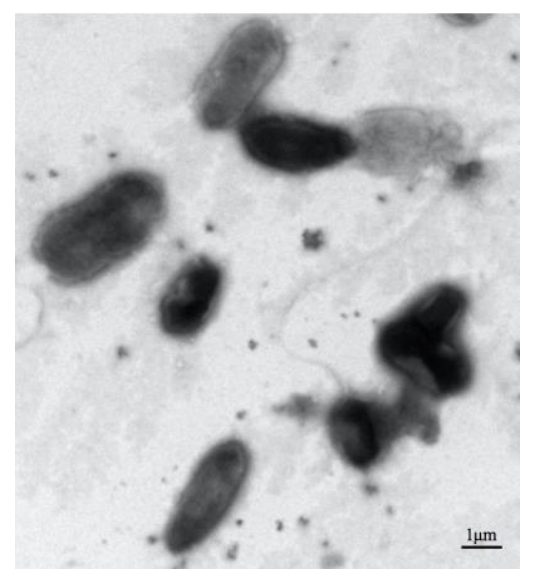

Fig 5. Negatively stained electron micrograph of $V$. natriegens from the tested P. trituberculatus showing its morphological characteristics. Scale bar, $1 \mu \mathrm{m}$. 


\section{Discussion}

$V$. natriegens was initially thought to be a non-pathogenic marine bacterium isolated from salt marshes (Payne et al., 1961). However, it has been verified as a local, highly prevalent, pathogen and has been isolated from aquatic animals and the environment. The pathogenicity of $V$. natriegens to aquatic animals seems to have been underestimated ( $\mathrm{Bi}$ et al., 2016; Deng et al., 2004; Li et al., 2009).

Traditionally, the detection of Vibrio species has been performed using a series of culture-based methods which involve enrichment, colony formation on selective agar medium, morphological, and biochemical tests. However, they are time-consuming and labor intensive. As the gold standard for Vibrio species detection, polymerase chain reaction (PCR), and real-time PCR are also impractical for in situ detection, due to the long operation time, and expensive, non-portable, instruments (Wang et al., 2017; Zhou et al., 2016; Garrido et al., 2012). In order to simply, rapidly, and accurately identify $V$. natriegens, a gyrB-LAMP method was developed. The gyrB gene that encodes the B subunit protein of bacterial DNA gyrase (topoisomerase type II) has been used for molecular identification (Yamamoto \& Harayama 1995). Both the conserved and the variable regions of this gene are suitable for the development of PCR primers (Kumar et al., 2006; Luo \& Hu 2008). The gyrB-targeted PCR methods have been developed for the identification of Vibrio (Kumar et al., 2006; Luo \& Hu 2008; Zhou et al., 2007; Cai et al., 2010). These studies demonstrate the preferred choice of the gyrB gene for molecular identification of closely related genomic species.

In this study, the established LAMP method was found to be a rapid, simple, and sensitive detection tool for species gyrB-targeted diagnosis of $V$. natriegens. It does not require gel electrophoresis, expensive equipment, and trained personnel. It is only necessary to maintain a constant temperature of $65^{\circ} \mathrm{C}$ for $1 \mathrm{~h}$ for the reaction. It can detect $V$. natriegens up to $1.32 \times 10^{-11}$ $\mathrm{fg} / \mathrm{mL}$ DNA template whereas using duplex PCR the detection of $V$. natriegens is possible up to $1.32 \times 10^{-5} \mathrm{fg} / \mathrm{mL}$ DNA template. All characterization mentioned above make LAMP a better choice than PCR and culture-based methods for the diagnosis of $V$. natriegens in cases where lower concentrations of bacteria are expected. Hence, the LAMP assay could be developed into a field test and made available to aquaculturists and producers in an effort to improve the prevention of $V$. natriegens.

In conclusion, the LAMP method described in this study represents a new sensitive, specific and rapid protocol for the detection of $V$. natriegens. It provides an important diagnostic tool for the detection of $V$. natriegens infection both in the laboratory and in the aquatic product market.

\section{Acknowledgements}

This work was supported by Natural science foundation of Jiangsu province of China (BK20140444, BK2012664), Open Project from Jiangsu Key Laboratory of Marine Biotechnology (HS2014013), a Project funded by the priority academic program development of Jiangsu higher education institutions (KYCX01) and Project from Huaihai Institute of Technology (KX11104).

\section{References}

Bi K.R., Zhang X.J., Yan B.L., Gao H., Gao X.J. and J.J. Sun, 2016. Isolation and molecular identification of Vibrio natriegens from diseased Portunus trituberculatus in China. $J$ World Aquacult Soc, 47(6), 854-861.

Cai S., Lu Y., Wu Z., Jian J., Wang B. and Y. Huang, 2010. Loop-mediated isothermal amplification method for rapid detection of Vibrio alginolyticus, the causative agent of vibriosis in mariculture fish. Letters in Appl Microbiol 50, 480-485.

Deng H., Chen Q., Shu H., Zhang D. and Z. Ma, 2004. The epizootic vibriosis in the larval bay scallop Argopecten irradians. J Dalian Fisheries University 19, 258-263.

Dhama K., Karthik K., Chakraborty S., Tiwari R., Kapoor S., Kumar A. and P. Thomas, 2014. Loop-mediated isothermal amplification of DNA (LAMP): A new diagnostic tool lights the world of diagnosis of animal and human pathogens: A review. Pakistan J Biol Sci 17(2), 151-166.

Garrido A., Chapela M., Ferreira M., Atanassova M., Fajardo P., Lago J., Vieites J. and A. Cabado, 2012. Development of a multiplex real-time PCR method for pathogenic Vibrio parahaemolyticus detection (tdh+ and trh+). Food Control 24, 128-135.

Kumar H., Parvathi A., Karunasagar I. and I. Karunasagar, 2006. A gyrB-based PCR for the detection of Vibrio vulnificus and its application for direct detection of this pathogen in oyster enrichment broths. Int J Food Microbiol 111, 216-220. 
Li G., Yan M., Sun J., Lin Z., Ma A. and W. Chang, 2009. dentification and biological characteristics of pathogen Vibrio natriegens from clam Meretrix meretrix. Progress in Fishery Sci 30, 103-109.

Liu Q., Li H., Wang Q., Liu P., Dai F. and J. Li, 2007. Identification and phylogenetic analysis of a strain of Vibrio alginolyticus, a pathogen in Portunus trituberculatus with toothpaste disease. Mar Freshwater Res 28, 9-13.

Luo P. and C. Hu, 2008. Vibrio alginolyticus gyrB sequence analysis and gyrB-targeted PCR identification in environmental isolates. Dis Aquat Organ 82, 209-216.

Lv J., Liu P., Gao B., Wang Y., Wang Z., Chen P. and J. Li, 2014. Transcriptome analysis of the Portunus trituberculatus: De novo assembly, growth-related gene identification and marker discovery. PLoS ONE 9(4): e94055. doi:10.1371/journal.pone.0094055

Notomi T., Okayama H., Masubuchi H., Yonekawa T., Watanabe K., Amino N. and T. Hase, 2000. Loop-mediated isothermal amplification of DNA. Nucl Acids Res 28(12), E63.

Payne W., Eagon R. and A. Williams, 1961. Some observations on the physiology of Pseudomonas Natriegens Nov. Spec. Antonie van Leeuwenhoek 27, 121-128.

Umar F., Rochman N., Wan R., Ahmadu S., Anas M., Sani A. and A. Atif, 2015. Loop-mediated isothermal amplification (LAMP), An innovation in gene amplification: bridging the gap in molecular diagnostics; A review. Indian J Sci Technol 8(17), doi:10.17485/ijst/2015/v8i17/55767.

Wan X., Shen H., Wang L. and Y. Cheng, 2011. Isolation and characterization of Vibrio metschnikovii causing infection in farmed Portunus trituberculatus in China. Aquacult Int 19, 351359.

Wang R., Xiao X., Chen Y., Wu J., Qian W., Wang L., Liu Y., Ji F. and J. Wu, 2017. A loopmediated, isothermal amplification-based method for visual detection of Vibrio parahaemolyticus within only $1 \mathrm{~h}$, from shrimp sampling to results. Anal. Methods 9, 1695-1701.

Weinstock M., Hesek E., Wilson C. and D. Gibson, 2016. Vibrio natriegens as a fast-growing host for molecular biology. Nature Methods 13, 849-851.

Yamamoto S. and S. Harayama, 1995. PCR amplification and direct sequencing of gyrB genes with universal primers and their application to the detection and taxonomic analysis of Pseudomonas putida strains. Appl Environ Microbiol 61, 1104-1109.

Yan, B., Qin G., Bao Z., Zhang X., Bi K. and L. Qin, 2010. Isolation and identification of Vibrio parahaemolyticus from diseased Portunus trituberculatus. Marine Sci Bull 29(5), 560-566.

Zhang X., Bai X., Yan B., Bi K. and L. Qin, 2014. Vibrio harveyi as a causative agent of mass mortalities of megalopa in the seed production of swimming crab Portunus trituberculatus. Aquacult Int 22(2), 661-672.

Zhou S., Gao Z., Zhang M., Liu D., Zhao X. and Y., Liu, 2016. Development of a quadruplex loop -mediated isothermal amplification assay for field detection of four Vibrio species associated with fish disease. Springerplus 5(1), 1104. doi:10.1186/s40064-016-2760-X.

Zhou S., Hou Z., Li N. and Q. Qin, 2007. Development of a SYBR Green I real-time PCR for quantitative detection of Vibrio alginolyticus in seawater and seafood. J Appl Microbiol 103, 18971906. 